\title{
What Contributes to Satisfaction with Care for Family Caregivers of Terminal Cancer Patients Admitted to General Wards? A Qualitative Study
}

\author{
Miki Morishita-Kawahara', ${ }^{1 *}$, Kiyoko Kamibeppu ${ }^{2}$ \\ ${ }^{1}$ Division of Gerontological Nursing, School of Nursing, Tokyo Women's Medical University, Tokyo, Japan \\ ${ }^{2}$ Department of Family Nursing, Graduate School of Health Sciences and Nursing, Faculty of Medicine, \\ The University of Tokyo, Tokyo, Japan \\ Email: *m-morisita@umin.ac.jp
}

How to cite this paper: Morishita-Kawahara, M. and Kamibeppu, K. (2020) What Contributes to Satisfaction with Care for Family Caregivers of Terminal Cancer Patients Admitted to General Wards? A Qualitative Study. Open Journal of Nursing, 10, 1081-1094.

https://doi.org/10.4236/ojn.2020.1011077

Received: October 25, 2020

Accepted: November 24, 2020

Published: November 27, 2020

Copyright $\odot 2020$ by author(s) and Scientific Research Publishing Inc. This work is licensed under the Creative Commons Attribution International License (CC BY 4.0).

http://creativecommons.org/licenses/by/4.0/

\begin{abstract}
Satisfaction with care is an important indicator for family caregivers of patients with terminal cancer and is linked to the quality of life. Despite this, few studies have examined the aspects of satisfaction with care of family caregivers of inpatients with cancer in general wards. This qualitative study aimed to elucidate the elements of satisfaction with the care that inpatients with terminal cancer in general wards and their family caregivers receive from medical staff, as perceived by the family caregivers. Semi-structured interviews were conducted with 10 family caregivers of inpatients with terminal cancer. Participants were asked about the care received until then from medical staff, the features of satisfactory care, and the aspects of care they felt were unsatisfactory or could become satisfactory with improvement. The data were analyzed with the content analysis method and the six categories were extracted. For family caregivers of inpatients with terminal cancer in general wards, along with the care identified as important in palliative care, the methods of alleviating symptoms and explaining the patient's condition were also important. The results highlight the importance of determining a patient-oriented approach and explanations together with each patient and family caregiver, based on an understanding of the long treatment process.
\end{abstract}

\section{Keywords}

Family Caregivers of Terminal Cancer Patients, General Wards, Qualitative Study, Satisfaction with Care 


\section{Introduction}

Cancer has a large impact on physical, psychological, and social factors in not only the patients themselves but also their family caregivers [1]. Consequently, quality of life (QOL) that is built on these elements also decreases [2] [3] [4]. In particular, family caregivers of patients with terminal cancer require complicated and advanced knowledge and must make decisions and face the death of a family member while supporting the patient's financial, social, and psychological needs [5] [6]. QOL during this stage drops to its lowest compared to the diagnosis stage or when the patient is still potentially curable [3] [7].

In Japan, the number of cancer patients spending their end of life at a palliative care unit or at home is increasing, but as of 2014 , about $80 \%$ still spend their final days on a general ward [8]. General wards have a wider variety of inpatients, from acute and chronic stage patients requiring treatment to those in terminal care focused on the alleviation of symptoms, and care and problems are complicated in these wards [9]. It has therefore been pointed out that palliative care is not as suitable in general wards as it is in palliative care units and hospice care [9]. As a result, family caregivers in general wards have a lower level of satisfaction [10] and even a lower QOL [11] [12] [13] than family caregivers of patients receiving hospice palliative care. Measures are therefore needed for family caregivers of inpatients with terminal cancer admitted to general wards. That said, one characteristic of family caregivers is that they wish for the patient's recovery even at the stage when no more treatments are available [14]. This means that it is highly likely that the family caregivers cannot accept facing the patient's death, and that a specific type of intervention is needed for general wards.

Satisfaction is defined to be comprised of expectations and individual needs [15]. Since death cannot be avoided in palliative care settings, one important outcome for both patients and family caregivers that has been researched is satisfaction with care [16] [17] [18] [19]. Past studies including our own research have demonstrated that satisfaction with care in family caregivers is not only one outcome, but also a factor related to QOL in family caregivers of cancer patients [3]. Increasing satisfaction with care is therefore essential to improve the QOL of family caregivers of patients with terminal cancer.

A review of the literature showed that important elements for determining satisfaction with care in the field of palliative care include accessibility, coordination, competence, communication, education, emotional support, personalization, and support of patients' decision-making [17]. Johnsen et al. conducted a survey of family caregivers of inpatients and outpatients with advanced cancer and found that satisfaction needed to be increased for the speed with which symptoms are treated and the time required to make a diagnosis [20]. Hannon et al. surveyed family caregivers of outpatients with advanced cancer and found that satisfaction needed to be increased for information regarding prognosis and pain management, and that satisfaction was lower than in patients for family inclusion in treatment/care decision and coordination of care, indicating a need 
for improvement [18]. Both of these studies focused on advanced cancer, and patients had a high level of activities of daily living, and so the studies cannot be said to clarify the type of satisfaction with care of family caregivers of patients with terminal cancer. This information can be examined from a literature review by Morita et al. carried out in Japan to create a care satisfaction scale for family caregivers of patients on palliative care units [16]. However, as shown above, considering the unique characteristics of family caregivers of patients spending their terminal stage on a general ward, it is uncertain whether the findings from the Morita et al. review can be applied in this case. Therefore, the aim of the present study was to elucidate the elements of care that inpatients with terminal cancer in general wards and their family caregivers receive from medical staff, as perceived by the family caregivers, with the purpose of improving the QOL of the family caregivers.

\section{Methods}

\subsection{Definition}

"Terminal" was operationally defined as the period aimed at alleviation of symptoms because the patient cannot undergo operative treatment or chemotherapy due to symptoms, and "care" was defined as actions for the patient and family caregivers by medical staff during hospital admission.

\subsection{Study Design and Participants}

A cross-sectional, qualitative study with semi-structured interviews was conducted from December 2012 to July 2013. Family caregivers of patients with terminal cancer were recruited from two general wards of two university hospitals in the Tokyo metropolitan area and its affiliated hospital. In Japan, death from digestive cancers represents more than $50 \%$ of total cancer deaths [21]. A high proportion of patients with digestive cancers along with those with non-curable or terminal stage cancers of other sites, experience nausea and vomiting (approximately 30\% - 70\%) [22], constipation (30\% - 90\%) [23], and malignant gastrointestinal obstruction ( $10 \%-50 \%$; occurring predominantly in patients with gastrointestinal and ovarian cancers) [24]. Consequently, patients in this study were recruited at the gastroenterological department ward.

Patients met the following criteria: 1) diagnosis of terminal digestive cancer, and 2) age $>20$ years. Eligibility criteria for family caregivers were: 1) a perceived by the patient as the primary caregiver, 2) age $>20$ years, 3) the survey participant was able to communicate, and 4) judged by the head nurse as physically and mentally capable of participating in the survey.

\subsection{Procedure}

The researcher and head nurse of the ward selected candidates who met the criteria. Next, the researcher gave an explanation to the patient and the family caregiver using a written explanation that was signed by both individuals. Family 
caregivers who provided consent were surveyed by face-to-face semi-structured interviews in private rooms with one of the authors. The interview content was recorded with an IC recorder with consent from the family caregiver being interviewed. After the interview, the family caregiver filled out a questionnaire about their characteristics. Lastly, the researchers examined the patient's medical records. Family caregivers received a book coupon worth 1,000 yen at the end of the interview as remuneration.

\subsection{Semi-Structured Interviews}

Family caregivers were given the definition of care at the start of the interview. An interview guide was developed to collect data from participants. The interview guide was consisted of the subscales of the Care Evaluation Scale (CES) [25]. Participants were asked about 1) the content of care they felt was satisfactory from their own awareness and 2) the content of care they felt was unsatisfactory and would be satisfactory with some improvement, concerning care received at the hospital. The CES is a scale for rating points considered important in palliative care concerning care items extracted from interviews of the bereaved family of patients who died in palliative care units [25]. Since care evaluation is linked to satisfaction with care [3], the subscales of the CES were used as the interview questions.

The CES has ten subscales: physical care provided by the physician, physical care provided by the nurse, psycho-existential care, help with decision-making for patient, help with decision-making for family, environment, Family burden, cost, availability of facilities, and coordination of care. In light of the definition of care used in the present study, all 10 topics aside from environment, cost, and availability of facilities were used as interview material.

\subsection{Analysis}

Analysis was based on Krippendorff's qualitative content analysis method [26]. This type of analysis defines the need to focus on context that generates a message. Context was prioritized in the present study as well, and the following procedure was used for analysis. First, verbatim transcripts were created and read repeatedly very carefully. Sentences related to satisfaction with care of family caregivers were extracted from the verbatim transcripts. Next, verbatim transcripts were divided by each semantic concept, paying attention to context, and they were summarized to create codes, without losing the original meaning. The codes created were grouped together into subcategories by similarity. Next, categories were created based on the similarity of subcategories. Three nurses specializing in oncology nursing and one gastroenterology specialist were consulted to determine the appropriateness of the subcategories and categories. In order to make the categorization more useful for care in clinical practice, we realized that the category names needed to have detailed descriptions, and not be too simple. All procedures performed in studies involving human participants were approved by the institutional review boards. 


\section{Results}

Twenty patients were selected from their medical records, and ten refused to participate. The main reason for refusing was that their family members were busy. Thus, 10 family caregivers were the subjects; none of the family caregivers refused to participate.

Nine of the ten family caregivers were women, and their mean age was 53.8 years. Six were spouses of the patient. Four of the patients were women, and the mean age was 69.5 years. A total of seven patients had a performance status of 3 or 4 , indicating need for nursing care in most activities (Table 1).

Interviews lasted a mean of $41.0 \pm 19.1$ minutes (range, 23 to 87 minutes). From the interview data, six categories and 29 subcategories were identified (Table 2). Categories are indicated in double angled brackets, and subcategories are indicated in square brackets below. Specific examples of comments by participants are indicated in italics, with supplementary information added by the researcher in parentheses to aid in comprehension.

1) "Medical staff gives quick, appropriate, and thoughtful care for distressing symptoms" [7 subcategories]

Regarding pain, [the patient is receiving appropriate care to alleviate pain] was given as a reason for satisfaction. In addition, dissatisfaction was felt when medical staff did not offer pain coping methods the patient desired, and the comment that [the family caregiver wants staff to use pain coping methods that match the patient's wishes] was given. In other words, participants pointed out the importance of not only relieving pain, but also of choosing pain alleviation methods based on the patient's wishes.

Table 1. Characteristics of subjects (family caregivers) and patients.

\begin{tabular}{|c|c|c|c|c|c|c|c|c|c|}
\hline \multirow{2}{*}{ ID } & \multicolumn{4}{|c|}{ FCs } & \multicolumn{5}{|c|}{ Patients } \\
\hline & Sex & Age & Education & Relationship & Sex & Age & Cancer & PS & Months \\
\hline 1 & $\mathrm{~F}$ & 43 & Higher education & Daughter & $\mathrm{F}$ & 70 & Pancreas & 3 & 2 \\
\hline 2 & $\mathrm{~F}$ & 28 & Higher education & Daughter & $\mathrm{F}$ & 63 & Pancreas & 4 & 5 \\
\hline 3 & $\mathrm{~F}$ & 57 & Higher education & Spouse & M & 60 & Gall bladder & 2 & 28 \\
\hline 4 & $\mathrm{~F}$ & 40 & Higher education & Daughter & M & 70 & Bile duct & 3 & 19 \\
\hline 5 & $\mathrm{~F}$ & 78 & Secondary Education & Spouse & $\mathrm{M}$ & 81 & Bile duct & 3 & 5 \\
\hline 6 & M & 62 & Higher education & Spouse & $\mathrm{F}$ & 65 & Bile duct & 1 & 12 \\
\hline 7 & $\mathrm{~F}$ & 46 & Higher education & Spouse & M & 61 & Large intestine & 3 & 48 \\
\hline 8 & $\mathrm{~F}$ & 53 & Higher education & Daughter & $\mathrm{M}$ & 85 & Papilla & 2 & 13 \\
\hline 9 & $\mathrm{~F}$ & 65 & Secondary Education & Spouse & $\mathrm{M}$ & 66 & Liver & 3 & 28 \\
\hline 10 & $\mathrm{~F}$ & 66 & Higher education & Spouse & $\mathrm{M}$ & 74 & Liver & 4 & 93 \\
\hline
\end{tabular}

F: female; M: male; FCs: family caregivers; PS: performance status; Months: months since diagnosis. Age is presented in years. A higher PS score indicates a worse general condition. 
Table 2. Satisfaction with care in family caregivers of inpatients with terminal cancer on general wards.

\begin{tabular}{ll}
\hline Categories & Subcategories \\
\hline
\end{tabular}

The patient is receiving appropriate care to alleviate pain.

Medical care being provided takes the patient's wishes regarding appearance into account.

Medical staff gives quick, appropriate, and thoughtful care for distressing symptoms
The family caregiver wants staff to provide hygiene care that matches the patient's symptoms and wishes.

The family caregiver wants staff to use pain coping methods that match the patient's wishes.

Nurses explain what side effects are expected to arise after testing or treatment ahead of time.

The family caregiver wants staff to carry out procedures that the patient wants.

Doctors carry out treatment and procedures quickly for the patient's distressing symptoms.

It is apparent that multiple staff are seeing the patient.

The family caregiver wants the palliative care team to directly explain to them changes in medications.

The family caregiver wants nurses to provide coordinated care.

Specialized staff works in the right space, cooperatively and consistently

They give explanations about the checkups or medicines, that are appropriate for each patient and also give information that includes treatment plans to patients and family caregivers

Nurses response promptly to nurse calls.

The family caregiver wants a doctor he or she can trust and ask questions.

The family caregiver wants what he or she has said to be conveyed accurately among the staff.

The family caregiver wants a straightforward explanation of the role of the palliative care team when it begins intervention.

The family caregiver wants a thorough explanation of the need for tests.

Explanations about medical narcotics to reduce the patient's anxiety were provided.

The patient's condition, information about the patient, and future prospects are conveyed accurately to the family.

The patient is given care in a manner that respects his or her feelings and wishes.

The family caregiver wanted detailed tests before diagnosis to be carried out quickly.

The patient was admitted to the hospital immediately after diagnosis.

They respect family caregivers agreement and understanding about the treatment process
The family caregiver wants staff to know the treatment process from diagnosis until the present.

The family caregiver wants information from past instances of hospital admission to be passed on. 


\section{Continued}

$\begin{aligned} & \text { The family caregiver wants the patient and family members } \\ & \text { to be consulted about medical prescriptions. }\end{aligned}$
$\begin{aligned} & \text { They encourage } \\ & \text { cooperative caring } \\ & \text { participation in hygiene care. }\end{aligned}$
$\begin{aligned} & \text { There are nurses the family caregiver can trust and consult. } \\ & \text { They give attention to mental } \\ & \text { aspects and daily life of } \\ & \text { family caregivers }\end{aligned}$
Attention is given to both the patient and family members.
Consideration is given to the situations of other families.
Family members are given consideration for their time.

ID2 said, "(About the fact that the patient was being switched from OxyContin to the Durotep patch from that day) I think it might have been easier for her (the patient) to have started on that first before taking the tablets. (So you wanted them to ask you first so you could choose?) Yes, exactly. Because I think it would be best to choose the method she wanted."

Aside from pain, three subcategories were generated concerning distressing symptoms such as constipation, ascites, abdominal bloating, and fever. ID7 said, "He (the patient) says that it is because of the water that he cannot eat, and so he is more interested in draining the water than discussing what to eat. As long as it is what he wants, I want the doctor to consider the possibility," expressing the desire for medical staff to consider the patient's wish to have his abdomen drained, for the subcategory [the family caregiver wants staff to carry out procedures that the patient wants].

2) "Specialized staff works in the right space, cooperatively and consistently" [7 subcategories]

From participants whose patient is receiving intervention from the palliative care team, comments were made about the prescriptions and explanation of the reason for intervention of the palliative care team. ID1 said, "(About the fact that the palliative care team always made their rounds in the morning) I did not meet them for the longest time, and then I met them the first time the other day when discussing changing hospitals. (Omission) My mother said that they were giving her medicine in this way from now on, and I kind of wished I had heard this for myself," expressing dissatisfaction. From this, [the family caregiver wants the palliative care team to directly explain to them changes in medications] was extracted. ID6 said, "Here, we listened to an explanation about palliative care the day after admission. The doctor explained it to us, and I felt like it was a little sudden. As soon as I heard the words 'palliative care,' all I could think was terminal care... Well I guess that the reality is that she is in the terminal stage." From this, [the family caregiver wants a straightforward explanation of the role of the palliative care team when it begins intervention] was extracted.

3) "They give explanations about the checkups or medicines that are appro- 
priate for each patient and also give information that includes treatment plans to patients and family caregivers" [4 subcategories]

For ID9 (recording rejection), the patient feels very tired because of the water retention in the abdomen, but he believes he has a kidney disease and still has hopes for recovery. Medical staff treats the patient with this in mind, and this creates satisfaction in the patient. Not only that, but the comment was also made that satisfaction of the patient also helps the family caregiver who is the subject of this study. From this, satisfaction was gained from the fact that [the patient is given care in a manner that respects his or her feelings and wishes]. ID5 said, "They thoroughly and accurately explain the situation to me and give me information, even if it is hard to say. Of course it is because of this that I know what we can and cannot do, and so I think it helps emotionally." On the other hand, ID6 was dissatisfied because the medical staff did not explain how much time the patient had left, saying, "The (voice breaks) situation was not very good, and she (my wife who was the patient) was there, so the doctor spoke positively and smoothly. So, I said I wanted to talk to the doctor one-on-one. We talked, and, as I thought, the doctor started discussing how much time she had left. If I had not asked, I would not have heard anything about how much time she had left." From this, [the patient's condition, information about the patient, and future prospects are conveyed accurately to the family] was extracted expressing both satisfaction and dissatisfaction.

4) 'They respect family caregivers' agreement and understanding about the treatment process" [4 subcategories]

Even in the terminal stage, it was important to be aware of not only the current distressing symptoms, but also the process in the stages prior to receiving treatment, indicated by the subcategories, [the family caregiver wanted detailed tests before diagnosis to be carried out quickly] and [the patient was admitted to the hospital immediately after diagnosis]. Also, ID7 said, "We couldn't do chemotherapy while he (the patient) had it (hepatitis). And of course, doctors and nurses change, and the new ones don't know about what happened before. One of them said to me that it would have been better for him to have started chemotherapy a little earlier... How much I actually wanted my husband to do chemotherapy... That bothered me... So, that kind of thing happens too," indicating dissatisfaction with an approach from medical staff who do not know the process during the treatment period. From this, [the family caregiver wants staff to know the treatment process from diagnosis until the present] was extracted.

5) "They encourage cooperative caring" [2 subcategories]

ID6 said, "The doctor told me about three days ago that she (the patient) was going to be given medication to activate her bowels, but she never got that medication. He never said anything to me about deciding not to prescribe it. I would feel more reassured if I heard the reason why they decided against it," expressing dissatisfaction because of the lack of explanation for family caregivers about prescriptions. From this, [the family caregiver wants the patient and fami- 
ly members to be consulted about medical prescriptions.] was extracted.

6) "They give attention to mental aspects and daily life of family caregivers" [5 subcategories]

Family caregivers want to tell nurses each time they have various problems or worries, and so [attention is given to both the patient and family members], [nurses appear to be listening to the family members] and [there are nurses the family caregiver can trust and consult] were extracted.

\section{Discussion}

First, the category "Medical staff gives quick, appropriate, and thoughtful care for distressing symptoms" indicates that family caregivers who were the subjects of the present study wanted quick alleviation of distressing physical symptoms. This has been clarified as one element of family caregivers' satisfaction with care for cancer patients staying in general wards [14], cancer patients staying in palliative care units [16], and cancer patients receiving care at home [27]. However, the subjects of the present study had hopes for not only quick alleviation of symptoms through symptomatic treatment, but also causal treatment for distressing physical symptoms, despite the patient being in the terminal stage. Moreover, it has been shown that family caregivers have a higher level of satisfaction as the number of options increases for the patient [28], and a low level of satisfaction concerning the provision of information about pain management methods [18]. In the present study as well, not only speed, but also methods were viewed as important, and the results pointed to the importance of individually-matched care that prioritizes provision of information and options in various areas of care, such as alleviation of symptoms and hygiene. In particular, despite quick pain relief being the most important aspect of care for family caregivers of patients with terminal cancer [29], family caregivers tend to have little knowledge about pain management [30]. It is therefore important to provide family caregivers with information needed for decision-making in which they participate.

Next, the category "specialized staff works in the right space, cooperatively and consistently" indicates that importance was placed on not only the approach of the primary physician or ward nurses, but also of the palliative care team. Past studies have shown that care from the palliative care team can alleviate symptoms and anxiety for the patient and reduce the risk of grief or poor mental condition in the family caregiver [14] [31], but this was the first study to specify that it also leads to family caregivers' satisfaction with care. For bereaved family members of cancer patients who died at home, referral of a palliative care specialist remained as a source of satisfaction [27]. However, family caregivers who were the subjects in the present study felt dissatisfaction from not receiving an explanation from the palliative care team staff directly, and they desired specific assistance, such as directly receiving an explanation. Accordingly, to enable active communication with the palliative care team staff, it may be important for 
ward staff to occasionally check family caregivers' availability and coordinate a visit with the palliative care team.

Regarding the category "they give explanations about the checkups or medicines that are appropriate for each patient and also give information that includes treatment plans to patients and family caregivers", a past study indicated that family caregivers' ability to evaluate how the patient's current condition and future prospects are conveyed to the patient is linked to family caregiver satisfaction [3]. In the present study as well, provision of information to patients and family caregivers including explanations about tests and medications and information about upcoming treatment plans was a source of satisfaction for family caregivers. This was consistent with a previous study of bereaved family members of cancer patients who had stayed on a palliative care ward [16]. However, regarding explanations for patients and family caregivers, the results of the present study indicated that family caregivers want to hear everything themselves, even if it is bad news, but they do not always want the patient's condition or progress of the disease to be conveyed accurately to the patient. In a past study, the hardest experience for family members of patients in the terminal stage was deciding whether or not to convey the truth [30]. It is therefore important for medical staff to first check with the family caregiver what the patient knows and their thoughts and discuss with the family caregiver how to approach the patient, creating a firm relationship between the medical staff and the family caregiver.

The most novel result obtained in the present study may be the category "they respect family caregivers' agreement and understanding about the treatment process". To the best of our knowledge, this was the first study to show that, even in the terminal stage when the treatment period is over, family caregivers were pleased when diagnosis and hospital admission went smoothly and felt dissatisfaction when staff did not know the treatment process up to that point. An important feature of general wards is that many patients receive uninterrupted care from diagnosis until end-of-life [32]. Accordingly, family caregivers may want medical staff to know the process taken up until the present and whether or not the patient was able to receive the desired treatment, for example. To improve the satisfaction with care, we considered that this category is important. Because it is not always easy to understand the whole process of the patient, medical staff should start to be concerned about it to improve the satisfaction with care.

We regarded the subcategories extracted in this study as items of satisfaction scale of care, and in our next study, we will quantitatively examine whether they are actually related to the QOL of family caregivers of terminal cancer patients admitted to a general ward. Another interesting research is to find factors which affect satisfaction with care.

The main limitation of this study was its small sample size. Furthermore, due to a research system for which we were unable to include other investigators, 
there was a need to conduct a survey focusing on the types of wards. As only the family caregivers of digestive cancer patients could be investigated, it was therefore, difficult to conclude that the subjects from this study comprise a sample representing family caregivers of terminal cancer patients admitted to a general ward. However, because we were able to target a wide range of cancer patients over a long period of time until now from diagnosis, we could investigate a wide variety of periods from diagnosis and the age of family caregivers. Furthermore, as the onset of digestive symptoms differs in many cancer patients [22] [23] [24], it was possible to clarify the details of care satisfaction from family caregivers within the limited scope of terminal care patients with digestive symptoms, since they visited relatively regularly during hospitalization in a general ward. It was difficult to ascertain when to submit a research request concerning family caregivers of terminal cancer patients, and recruiting patients was difficult. Moreover, in addition to cooperating with the clinical staff, it was necessary to also consider the balance of patient recruitment along with appropriate ethical considerations.

\section{Conclusion}

In the present study, the type of satisfaction with care of family caregivers of inpatients with terminal cancer on general wards was clarified. As a result, regarding alleviation and explanation of symptoms, the results highlight the importance of a patient-oriented approach and explanations together with each patient and family caregiver, based on an understanding of the long process up to that point. Approaches in general wards should therefore take into account not only the terminal stage but also the diagnosis and treatment stage.

\section{Funding}

This research did not receive any specific grant from funding agencies in the public, commercial, or not-for-profit sectors.

\section{Author Contributions}

Conceptualization, M.M.K. and K.K.; methodology, M.M.K.; analysis, M.M.K.; investigation, M.M.K.; writing-original draft preparation, M.M.K.; writingreview and editing, K.K.; supervision, K.K.

\section{Conflicts of Interest}

The authors declare no conflicts of interest regarding the publication of this paper.

\section{References}

[1] Stenberg, U., Ruland, C.M. and Miaskowski, C. (2010) Review of the Literature on the Effects of Caring for a Patient with Cancer. Psychooncology, 19, 1013-1025. https://doi.org/10.1002/pon.1670 
[2] Testa, M.A. and Simonson, D. (1996) Assessment of Quality of Life Outcomes. New England Journal of Medicine, 334, 835-840. https://doi.org/10.1056/NEJM199603283341306

[3] Morishita, M. and Kamibeppu, K. (2014) Quality of Life and Satisfaction with Care among Family Caregivers of Patients with Recurrent or Metastasized Digestive Cancer Requiring Palliative Care. Supportive Care in Cancer, 22, 2687-2696. https://doi.org/10.1007/s00520-014-2259-3

[4] Song, J.I., Shin, D.W., Choi, J.Y., Kang, J., Baik, Y.J., Mo, H., Park, M.H., Choi, S.E., Kwak, J.H. and Kim, E.J. (2011) Quality of Life and Mental Health in Family Caregivers of Patients with Terminal Cancer. Supportive Care in Cancer, 19, 1519-1526. https://doi.org/10.1007/s00520-010-0977-8

[5] Hudson, P. (2004) Positive Aspects and Challenges Associated with Caring for a Dying Relative at Home. International Journal of Palliative Nursing, 10, 58-65. https://doi.org/10.12968/ijpn.2004.10.2.12454

[6] Rabow, M.W., Hauser, J.M. and Adams, J. (2004) Supporting Family Caregivers at the End of Life: “They Don't Know What They Don't Know”. JAMA, 291, 483-491. https://doi.org/10.1001/jama.291.4.483

[7] Grunfeld, E., Coyle, D., Whelan, T., Clinch, J., Reyno, L., Earle, C.C., Willan, A., Viola, R., Coristine, M., Janz, T. and Glossop, R. (2004) Family Caregiver Burden: Results of a Longitudinal Study of Breast Cancer Patients and Their Principal Caregivers. CMAJ, 170, 1795-1801. https://doi.org/10.1503/cmaj.1031205

[8] White Paper on Hospice and Palliative Care 2016. https://www.hospat.org/assets/templates/hospat/pdf/hakusyo_2016/2016_2.pdf

[9] Terakado, A. and Matsushima, E. (2015) Work Stress among Nurses Engaged in Palliative Care on General Wards. Psychooncology, 24, 63-69. https://doi.org/10.1002/pon.3584

[10] Miceil, P.J. and Mylod, D.E. (2003) Satisfaction of Families Using End-of-Life Care: Current Successes and Challenges in the Hospice Industry. American Journal of Hospice and Palliative Medicine, 20, 360-370. https://doi.org/10.1177/104990910302000510

[11] Wright, A.A., Zhang, B., Ray, A., Mack, J.W., Trice, E., Balboni, T., Mitchell, S.L., Jackson, V.A., Block, S.D., Maciejewski, P.K. and Prigerson, H.G. (2008) Associations between End-of-Life Discussions, Patient Mental Health, Medical Care near Death, and Caregiver Bereavement Adjustment. JAMA, 300, 1665-1673. https://doi.org/10.1001/jama.300.14.1665

[12] Wright, A.A., Keating, N.L., Ayanian, J.Z., Chrischilles, E.A., Kahn, K.L., Ritchie, C.S., Weeks, J.C., Earle, C.C. and Landrum, M.B. (2016) Family Perspectives on Aggressive Cancer Care near the End of Life. JAMA, 315, 284-292. https://doi.org/10.1001/jama.2015.18604

[13] Ornstein, K.A., Aldridge, M.D., Garrido, M.M., Gorges, R., Meier, D.E. and Kelley, A.S. (2015) Association between Hospice Use and Depressive Symptoms in Surviving Spouses. JAMA Internal Medicine, 175, 1138-1146.

https://doi.org/10.1001/jamainternmed.2015.1722

[14] Yang, G.M., Ewing, G. and Booth, S. (2012) What Is the Role of Specialist Palliative Care in an Acute Hospital Setting? A Qualitative Study Exploring Views of Patients and Carers. Palliative Medicine, 26, 1011-1017. https://doi.org/10.1177/0269216311425097

[15] Aspinal, F., Addington-Hall, J., Hughes, R. and Higginson, I.J. (2003) Using Satisfaction to Measure the Quality of Palliative Care: A Review of the Literature. Journal 
of Advanced Nursing, 42, 324-339.

https://doi.org/10.1046/j.1365-2648.2003.02624.x

[16] Morita, T., Chihara, S. and Kashiwagi, T. (2002) A Scale to Measure Satisfaction of Bereaved Family Receiving Inpatient Palliative Care. Palliative Medicine, 16, 141-150. https://doi.org/10.1191/0269216302pm514oa

[17] Dy, S.M., Shugarman, L.R., Lorenz, K.A., Mularski, R.A. and Lynn, J. (2008) A Systematic Review of Satisfaction with Care at the End of Life. Journal of the American Geriatrics Society, 56, 124-129. https://doi.org/10.1111/j.1532-5415.2007.01507.x

[18] Hannon, B., Swami, N., Krzyzanowska, M.K., Leighl, N., Rodin, G., Le, L.W. and Zimmermann, C. (2013) Satisfaction with Oncology Care among Patients with Advanced Cancer and Their Caregivers. Quality of Life Research, 22, 2341-2349. https://doi.org/10.1007/s11136-013-0371-3

[19] Heyland, D.K., Frank, C., Tranmer, J., Paul, N., Pichora, D., Jiang, X. and Day, A.G. (2009) Satisfaction with End-of-Life Care: A Longitudinal Study of Patients and Their Family Caregivers in the Last Months of Life. Journal of Palliative Care, 25, 245-256. https://doi.org/10.1177/082585970902500402

[20] Johnsen, A.T., Ross, L., Petersen, M.A., Lund, L. and Groenvold, M. (2012) The Relatives' Perspective on Advanced Cancer Care in Denmark. A Cross-Sectional Survey. Supportive Care in Cancer, 20, 3179-3188. https://doi.org/10.1007/s00520-012-1454-3

[21] Center for Cancer Control and Information Services, National Cancer Center, Japan. (2020) http://ganjoho.jp/reg_stat/statistics/stat/summary.html

[22] Davis, M.P. and Walsh, D. (2000) Treatment of Nausea and Vomiting in Advanced Cancer. Supportive Care in Cancer, 8, 444-452.

https://doi.org/10.1007/s005200000151

[23] Larkin, P.J., Sykes, N.P., Centeno, C., Ellershaw, J.E., Elsner, F., Eugene, B., Gootjes, J.R., Nabal, M., Noguera, A., Ripamonti, C., Zucco, F. and Zuurmond, W.W. (2008) The Management of Constipation in Palliative Care: Clinical Practice Recommendations. Palliative Medicine, 22, 796-807. https://doi.org/10.1177/0269216308096908

[24] Ripamont, C. and Bruera, E. (2002) Palliative Management of Malignant Bowel Obstruction. International Journal of Gynecological Cancer, 12, 135-143.

[25] Morita, T., Hirai, K., Sakaguchi, Y., Maeyama, E., Tsuneto, S. and Shima, Y. (2004) Measuring the Quality of Structure and Process in End-of-Life Care from the Bereaved Family Perspective. Journal of Pain and Symptom Management, 27, 492-501. https://doi.org/10.1016/j.jpainsymman.2003.10.014

[26] Krippendorff, K. (1980) Content Analysis: An Introduction to Its Methodology. Sage Publications, Beverly Hills.

[27] Kristjanson, L.J. (1993) Validity and Reliability Testing of the FAMCARE Scale: Measuring Family Satisfaction with Advanced Cancer Care. Social Science \& Medicine, 36, 693-701. https://doi.org/10.1016/0277-9536(93)90066-D

[28] Fakhoury, W., McCarthy, M. and Addington-Hall, J. (1996) Determinants of Informal Caregivers' Satisfaction with Services for Dying Cancer Patients. Social Science \& Medicine, 42, 721-731. https://doi.org/10.1016/0277-9536(95)00198-0

[29] Fakhoury, W.K., McCarthy, M. and Addington-Hall, J. (1997) The Effects of the Clinical Characteristics of Dying Cancer Patients on Informal Caregivers' Satisfaction with Palliative Care. Palliative Medicine, 11, 107-115.

https://doi.org/10.1177/026921639701100204 
[30] Huang, H.L., Chiu, T.Y., Lee, L.T., Yao, C.A., Chen, C.Y. and Hu, W.Y. (2012) Family Experience with Difficult Decisions in End-of-Life Care. Psychooncology, 21, 785-791. https://doi.org/10.1002/pon.3107

[31] Kang, J., Shin, D.W., Choi, J.E., Sanjo, M., Yoon, S.J., Kim, H.K., Oh, M.S., Kwen, H.S., Choi, H.Y. and Yoon, W.H. (2013) Factors Associated with Positive Consequences of Serving as a Family Caregiver for a Terminal Cancer Patient. Psychooncology, 22, 564-571. https://doi.org/10.1002/pon.3033

[32] Friedrichsen, M., Hajradinovic, Y., Jakobsson, M., Milberg, P. and Milberg, A. (2017) Palliative Care Consultation Team on Acute Wards-An Intervention Study with Pre-Post Comparisons. Supportive Care in Cancer, 25, 371-380.

https://doi.org/10.1007/s00520-016-3406-9 